\title{
De quoi Europe est-il le nom ? Enjeux et usages argumentatifs de la polyréférentialité
}

What is Named "Europe"? The Uses of Multireference and its Argumentative Stakes

Julien Auboussier

\section{(2) OpenEdition}

\section{Journals}

Édition électronique

URL : http://journals.openedition.org/aad/2216

DOI : 10.4000/aad.2216

ISSN : $1565-8961$

Éditeur

Université de Tel-Aviv

Référence électronique

Julien Auboussier, « De quoi Europe est-il le nom ? Enjeux et usages argumentatifs de la

polyréférentialité », Argumentation et Analyse du Discours [En ligne], 17| 2016, mis en ligne le 15 octobre 2016, consulté le 23 septembre 2019. URL : http://journals.openedition.org/aad/2216 ; DOI : 10.4000/ aad.2216

Ce document a été généré automatiquement le 23 septembre 2019

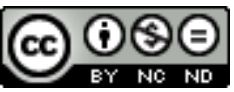

Argumentation \& analyse du discours est mis à disposition selon les termes de la licence Creative Commons Attribution - Pas d'Utilisation Commerciale - Pas de Modification 4.0 International. 


\title{
De quoi Europe est-il le nom? Enjeux et usages argumentatifs de la polyréférentialité
}

\author{
What is Named "Europe"? The Uses of Multireference and its Argumentative \\ Stakes
}

Julien Auboussier

\section{Introduction}

1 La valeur géographique du nom propre Europe est évidente. Bien que mal délimité, l'espace ainsi nommé jouit d'un prédicat de réalité et d'une fixation référentielle qui lie le signe à l'objet du monde. Pour autant, le lecteur de presse, notamment, s'aperçoit au quotidien que Europe peut avoir divers référents qui l'éloignent de son potentiel toponymique pour revêtir de multiples sens. Dès lors, nous proposons d'adopter « une approche discursive du nom propre » (Lecolle, Paveau et Reboul-Touré 2009). En effet, si Europe ne peut se réduire à sa seule valeur géographique, il est nécessaire d'en interroger à la fois la polyréférientialité, pour rendre compte de «la souplesse du toponyme » (Paveau 2008), et les potentialités argumentatives que son inscription en discours autorise. Il ne s'agit donc pas seulement de lister les référents possibles d'Europe dans les figures rhétoriques routinisées de l'écriture journalistique, mais aussi de relever les enjeux politiques de la nomination dans le cadre des discours rapportés au sein de la presse française.

2 Si la dénomination s'inscrit dans la problématique du signe qui pointe quelque chose et interroge donc la référence, la nomination constitue, quant à elle, « un acte de parole contextualisé » (Siblot 2001). En proposant d'interroger le nom propre Europe, nous associons donc l'étude du parcours référentiel qui l'associe à une chose et l'actualisation de son sens en discours. «Europe » fait partie du système de la langue, désignateur individualisant, mais son fonctionnement s'inscrit dans les dynamiques du discours. Son référent s'actualise dans l'énonciation, rendant ainsi compte d'une part 
de "l'épaisseur sémantique » du nom propre (Barthes 1972) et de son aptitude à la polyréférentialité (Cislaru 2005), d'autre part, de la "production contextuelle et interdiscursive du sens » et des potentialités signifiantes du nom propre (Siblot $2001: \S$ 2.1). Marie-Anne Paveau relève : "Que le nom propre ait un sens, et même plusieurs, est en effet de l'ordre de l'évidence... hors de la linguistique "savante", où cette question est constamment en débat» (2008: 24)1. Ces interrogations sont largement alimentées depuis une dizaine d'années par l'étude de l'onomastique (Boyer, Paveau 2008), des noms de lieux (Draga 2010), des noms de villes (Yasri-Labrique 2008) ou encore des noms de pays (Cislaru 2005, 2008). Par ailleurs, la dimension sémantique est au centre des théories du «nom propre modifié » (Noailly 1999, Leroy 2005) par un déterminant qui participe à sa polyréférentialité et à sa polysémie. Dans une perspective référentialiste, le nom propre modifié ne désigne plus directement son référent initial mais soit un autre référent soit "une facette de ce référent » (Leroy 2004 : 49). Si le nom propre modifié n'est pas au centre de ce travail, reste que nous le rencontrerons nécessairement et l'analyserons au regard de ses potentialités pragmatiques dans le travail d'argumentation politique.

3 L'étude est exploratoire et s'appuie sur des données recueillies depuis la base de données Europresse dans les trois quotidiens français dits « de référence » c'est-à-dire Le Monde, Libération et Le Figaro (version papier et numérique). Les extraits cités sont autant que possible contextualisés afin de permettre une meilleure évaluation des enjeux politiques de la nomination. Dans la dernière partie, notre attention se concentre sur le commentaire médiatique qui se déploie lors d'une séquence d'actualité : le conflit en Ukraine. Un corpus clos a alors été constitué avec les tribunes et les éditoriaux - genres à «énonciation subjectivisée " (Moirand 2000) - relatifs aux événements ukrainiens publiés dans les trois mêmes quotidiens ainsi que dans l'hebdomadaire Marianne.

4 Notre propos suivra, en quelque sorte, le parcours référentiel de la dénomination : nous partons du mythe de l'enlèvement d'Europe (nom propre prototypique), référent initial dont les réapparitions en contexte médiatique méritent d'être interrogées; nous abordons ensuite l'opacité référentielle liée aux usages métonymiques routinisés du nom propre dans le récit de l'actualité et les enjeux pragmatiques de la personnalisation ; enfin, les usages modifiés qui jouent de la polyréférentialité dans une visée argumentative et contribuent à l'actualisation du sens sont interrogés au regard des enjeux politiques auxquels ils répondent.

\section{Du mythe à la géographie. L'abandon du référent initial}

5 De quoi Europe est-il originellement le nom ? Dans la mythologie grecque, «Europe » nomme une jeune princesse phénicienne. Fille d'Agénor, roi de Phénicie, Europe est victime des appétits de Zeus. Du haut de l'Olympe, ce dernier en tombe éperdument amoureux et décide de la conquérir en usant de la ruse. Pour échapper à son épouse, il se métamorphose en un magnifique taureau blanc pour s'approcher d'Europe. D'abord effrayée, la princesse s'approche et, surmontant sa peur, elle le caresse et finalement s'assoit sur son dos. A cet instant précis, ce Zeus transformé en Taureau, s'élance vers la mer, s'enfonce dans les flots malgré les cris de désespoir de la jeune princesse et la mène jusqu'en Crète. C'est « l'enlèvement d'Europe». 
6 Europe revêt ici les attributs formels du nom propre: majuscule et absence de déterminant. Désignant, de manière rigide, un individu unique par un lien qui relève de la convention et non du sens, Europe nomme et maintient une singularité sans la décrire. Etymologiquement, le référent initial associé à Europe est donc une princesse et l'association est issue d'un « acte de baptême » qui fige la référence (Kleiber 1984).

7 Gary-Prieur distingue « le référent initial associé à un Np par un acte de baptême et le référent discursif qui peut être attribué à ce même $\mathrm{Np}$ du fait de sa position, dans un discours donné, dans un contexte incompatible avec l'instruction référentielle qui constitue le sens en langue de ce Np (1994: 35). Il semble difficile de faire de cette première nomination le référent initial des référents discursifs identifiés dans les discours contemporains, médiatiques ou politiques. Ceci ne signifie pas, néanmoins, la disparition totale $d u$ référent initial. Europe,comme nom propre prototypique, réapparait par exemple quand la presse présente les nouveaux billets mis en circulation par la Banque centrale européenne (de 2013 à 2016) et sur lesquels figure la princesse Europe. Mais, surtout, la mobilisation du référent initial peut servir une visée de persuasion et alimenter l'argumentation. Le locuteur peut ainsi proposer une comparaison entre le rapt d'Europe et la situation économique ou politique actuelle. Ainsi, dans une tribune publiée dans Le Figaro, Renaud Girard revendique un " protectionnisme européen raisonné » en proposant un tel parallèle. Dans cet extrait, comme dans d'autres, c'est la vulnérabilité de la jeune princesse que le locuteur retient du mythe pour ensuite l'associer à la faiblesse de l'Union européenne face aux intérêts extra-communautaires :

(1) Jadis enlevée par Zeus, déguisé en taureau, notre belle Europe s'est-elle faite, au tournant du millénaire, violée par le marchand chinois? Le terme est un peu fort, dans la mesure où, à Bruxelles, la nouvelle Sidon, notre princesse avait eu tout le loisir de réfléchir avant de décider à se donner aux chinois. Mais le moins que l'on puisse dire est que, dans cette relation, Europe ne s'est pas montrée très farouche (Le Figaro, 03-04-2016).

8 Si Europe nomme originellement un individu, la dénomination revêt rapidement une autre valeur jusqu'à perdre le lien qui l'unissait à son référent initial. Pour Tournier, la première dénomination géographique d'Europe date du $7^{\mathrm{e}}$ siècle av. J.-C. et désigne "tout ce qui, pour les Grecs, n'était pas "barbaros" c'est-à-dire étranger bredouillant, sans culture ni langue » (Tournier $2002: 87$ ). Pour autant, il faut préciser, à la suite de l'historien John Hale, que ce n'est qu'à la Renaissance que le mot Europe entre dans le langage courant et se dote d'un cadre de référence, notamment, appuyé, sur la cartographie (Hale 1993). A partir du 16 ${ }^{\mathrm{e}}$ siècle, les dérivés issus de la base lexicale Europe - notamment européen- fondent la référence à une identité ethnique et/ou culturelle (Luxardo, Richard, Steuckardt 2015: 165). En fait, comme le relève Guilhaumou (2015: 21), Europe est alors quasi-synonyme de chrétienté : «L'Europe est alors définie dans un cadre toujours médiéval mais ce cadre culturel est désormais renvoyé à une unité territoriale, et non plus à une abstraite Respublica Christiana extensible à l'ensemble de la sphère terrestre ». Les limites de l'espace nommé ne cesseront ensuite d'évoluer au gré des conquêtes, des invasions et « des progrès de la civilisation $»^{2}$. Dès lors, l'Europe se définit dans le rapport qu'elle entretient à l'ailleurs : la dénomination devient géographique. L'existence des mots offre aux objets qu'ils nomment une réalité séparée : la dénomination Europe distingue ainsi un fragment du monde. Si l'incertitude et l'instabilité des limites de l'espace ainsi nommé persistent jusqu'à aujourd'hui, elles ne nuisent aucunement au prédicat de réalité (« cela est !»). 
9 A ce titre, de la mythologie à la géographie (de «Europe» à "l'Europe »), la dénomination se dote d'un déterminant qui permet de classer la forme dans la catégorie des noms propres modifiés. Comme pour les noms de pays (Cislaru 2006), et contrairement au nom propre prototypique, les noms de continent ont en effet pour spécificité morphosyntaxique d'être accompagnés d'un article défini dit lexical: « L'article défini est associé au nom [propre] dans le système de la langue, en vertu du mode de référence propre à ces noms, et non dans l'énoncé, comme c'est le cas pour les autres noms précédés à l'occasion d'un article défini " (Gary-Prieur 1994 : 229). L'unicité du référent est ainsi "garantie indépendamment de toute variation des univers de croyances $»(\text { idem })^{3}$.

La dimension locative de la dénomination est forte puisqu'elle pointe un fragment situé de la surface du monde. Signifié et signifiant désignent ainsi un référent (assez) clairement identifié. Cette valeur géographique alimente de nombreux usages de la dénomination l'Europe dans la presse au sein desquels le flou sur l'espace désigné ne vient pas gêner la compréhension du lecteur :

(2) Pegida en Allemagne, le Front national en France, Aube dorée en Grèce, le FPÖ en Autriche... Les extrêmes droites - au pluriel - ne cessent d'agiter le champ politique en Europe depuis plusieurs années. [...] Et leur dynamique ne devrait pas être freinée dans les années à venir. «Avec la conjonction de ces crises, l'extrême droite ne saurait mourir en Europe. Comme elle fait partie de notre histoire, elle participera de notre avenir ", résument les auteurs [J.-Y. Camus et N. Lebourg] (Le Monde, 7-12-2015).

(3) À l'heure où la peur du terrorisme djihadiste se répand dans toute l'Europe, un germe d'espoir s'épanouit au cœur de la Suisse neutre, un espace de dialogue. Cinq lieux de culte sont réunis sous un même toit (Le Monde, 11-07-2015).

11 En (2) et (3), Europe a une valeur distinctive (vis-à-vis du reste du monde) qui identifie un référent unique. Les prépositions en et dans affirment la valeur locative de la dénomination. Dans les deux cas, au regard du contexte, la recherche du référent initial ne peut mener qu'à la géographie et non, dans la dynamique métonymique que l'on abordera ensuite, à l'Union européenne. En effet, en (2), la montée des partis d'extrême droite ne se limite pas aux pays membres (à l'exemple de la Suisse et de la Norvège citées dans l'article). De même, en (3), le référent initial ne peut être que géographique : «la peur du terrorisme djihadiste " se déploie en effet dans un espace géographique dont les limites ne peuvent pas être assimilées à celles des institutions politiques européennes. Dans ces deux énoncées, représentatifs de nombreux usages, le prédicat de signification « continent » est attribué à Europe. C'est l'emploi correspondant à la première définition qu'en donnent les dictionnaires usuels.

\section{De la géographie à l'institution politique}

\subsection{Métonymie et routines journalistiques}

12 Dans la presse, "l'Europe " désigne très souvent l'Union européenne et ses composantes, dirigeants ou membres ${ }^{4}$. Dès lors, la valeur géographique du terme disparaît dans une dynamique métonymique qui incite à la recherche d'un référent sous-jacent. Prenons en exemple un enchaînement titre/chapeau/début d'article. L'article porte principalement sur le projet de directive PNR (Passenger Name Record) dans le contexte des attentats parisiens de janvier puis novembre 2015 : 
(4) L'Europe résolue à agir

A la suite de la tuerie au siège de Charlie Hebdo et à l'Hyper Cacher, Bruxelles avait vivement réagi disant sa volonté d'agir.

La Commission avait promis de réactiver une proposition de directive PNR et de renforcer la coopération avec les géants du Web pour faire la chasse aux sites

djihadistes (Le Monde, 20-11-2015).

13

( paradigne désignationn L'Europe s'inscrit aux côtés de Bruxelles et la Commission. Clairement, dans cet exemple, on ne peut assigner de référence précise à la dénomination l'Europe qu'au terme de la lecture des premières lignes de l'article c'est-à-dire qu'en prenant en compte un cotexte élargi au transphrastique. Ici, «l'Europe» est mis pour la Commission (européenne) qui apparaît comme le référent sous-jacent. Le caractère substituable des termes offre un indice de coréférencement, notamment, comme ici, dans la succession titre/chapeau/article (Krieg-Planque 2008).

La substitution, comme " transfert sémantico-référentiel affectant le mot ou le syntagme» (Bonhomme 1987), répond à une pratique de la métonymie habituelle dans l'écriture de presse: le lieu pour les dirigeants qui y siègent, le nom de ville pour l'institution, l'institution pour le lieu qui l'abrite... (Lecolle 2001). Autrement dit, la fixation référentielle entre l'Union européenne et la dénomination l'Europe ne tient pas d'un acte de nomination effectif. Pour autant, comme le note Kleiber (2001), revenant sur ses premiers travaux, la dénomination peut exister sans «acte de baptême » à condition que la relation entre une chose $\mathrm{x}$ et un signe $\mathrm{X}$ résulte d'une «habitude associative» qui résulte, en l'occurrence, à la fois d'une routine journalistique (et, du côté du lecteur, d'une routine interprétative).

Le processus de substitution fonctionne en fait au sein d'un paradigme désignationnel élargi à l'ensemble des institutions siégeant dans la capitale belge et à leurs membres : l'Europe > Union européenne > Bruxelles > Commission européenne (ou les commissaires) / Conseil des ministres (ou les ministres) / Conseil de l'Europe(ou ses membres). Cela contribue à confirmer que «l'indétermination référentielle est souvent un fait significatif de la métonymie, du moins dans le discours journalistique » (Lecolle 2001 : 103). La substitution ne répond pas ici à une visée argumentative et ne génère pas d'effets rhétoriques importants. Pour autant, cela ne signifie pas qu'elle ne revêt aucun enjeu : à la dénomination métonymique répond ainsi une pluralité référentielle qui participe à une représentation unifiée de l'Europe faisant fi de la réalité conflictuelle de l'Union européenne (notamment des clivages entre les députés strasbourgeois et les commissaires bruxellois) et des multiples médiations de l'Europe politique ${ }^{5}$.

\subsection{Personnification et routines journalistiques}

Comme dans le cas des noms de pays, l'emploi métonymique encourage l'attribution à l'Europe d'un statut d'actant dans le récit de l'actualité. Les occurrences actantielles sont courantes et peuvent, parfois, s'apparenter à des personnifications ${ }^{6}$. Personnifiée, « espèce d'être réel ou physique, doué de sentiment et de vie " (Lakoff, Johnson 1985), l'Europe n'est plus réductible à ses caractéristiques référentielles (être composé de diverses institutions et de leurs membres) mais profite d'une vie propre. "Le procédé peut renvoyer à la volonté de la part du journaliste de rendre vivante la situation qu'il

Argumentation et Analyse du Discours, 17 | 2016 
décrit : en personnifiant des entités politiques, il rend plus accessible l'information par le lecteur » (Develotte 2000, 27). Un seul exemple :

(5) Pendant que l'Europe s'aigrit et se ferme, l'Allemagne a analysé son déficit en main-d'œuvre et dépassé l'obstacle par l'ambition d'un grand plan d'accueil comparable à celui mis en place lorsqu'il avait fallu réaliser l'unification avec l'Est, après 1990 (Le Monde, 14-12-2015).

En position sujet, l'Europe se voit attribuer des états d'âme et des actes (qui dépassent les actes de langage propres aux institutions politiques du type "ratifier», " condamner", "déclarer»). Dans les discours assumés par le journaliste archiénonciateur, la personnification relève, comme la métonymie, d'une routine qui participe à la vitalité du style journalistique et au souhait de rendre accessible la complexité du processus de décision communautaire.

Dans le discours rapporté du personnel politique (principalement chez les opposants à l'intégration), la personnification revêt d'autres enjeux et participe clairement aux arguments de causalité en facilitant le biais de complaisance. «Inhérent à tout discours argumentatif [et] constituant ainsi une stratégie centrale de toute rhétorique politique " (Gosselin 1995 : 155), le biais de complaisance désigne l'attitude de l'homme politique qui s'attribue, à lui-même et à ses qualités personnelles, les succès constatés et qui explique et impute les échecs à des facteurs externes, en l'occurrence à l'Europe. Personnalisée, l'Europe vient souvent s'inscrire dans un schéma actanciel qui l'oppose au peuple et à ses intérêts (dont le locuteur se présente comme le seul garant) :

(6) A la tribune, Marine Le Pen se lance dans une longue diatribe contre l'Union européenne accusée de tous les maux : «L'Europe martyrise les peuples, mutile nos libertés, dilue les identités et s'attaque à l'indispensable souveraineté des nations. » (Le Monde.fr, 12-02-2015).

Le journaliste précise le référent désigné dans la diatribe de Marine Le Pen qui, elle, s'en tient à l'ambiguë dénomination Europe. L'on remarque surtout la succession des verbes d'action à forte tonalité axiologique (martyriser, mutiler, diluer, s'attaquer) associés à Europe. La personnification s'inscrit là dans un appel à l'émotion que l'« agencement rythmique » du propos vient clairement renforcer (Koren 2004). Dans le discours rapporté de $\mathrm{M}$. Le Pen, si le référent géographique ne s'efface pas complétement, il apparaît très secondaire face à la référence à l'institution politique.

Les opposants à l'intégration, dans leurs discours, construisent ainsi souvent l'Europe non pas comme un espace politique conflictuel mais nomme une entité agissante, le plus souvent en sous-main, dont il s'agit de dévoiler le mépris du peuple, l'opacité des décisions, la corruption des acteurs. La polyréférentialité de la dénomination permet de brosser le portrait flou d'un adversaire (parle-t-on de l'Union européenne ? de la seule Commission ? du Parlement ${ }^{7}$ ?); elle inscrit ainsi l'Europe dans un paradigme événementiel où figurent d'ores et déjà l'élite, l'establishment et autres dénominations dont l'ambiguïté référentielle participe à l'efficacité pragmatique des discours populistes (Charaudeau 2011). 


\section{Qualifier l'Europe. De la dénomination à la désignation axiologique}

\subsection{Au-delà de la désignation, la caractérisation}

Au sein du discours rapporté, la dénomination sert de base à un nombre conséquent de syntagmes nominaux dans lesquels, à la référence inscrite dans le nom propre, s'ajoute l'expression de la caractérisation. L'on passe alors de la dénomination à des désignations descriptives qui se répartissent entre celles qui qualifient l'Europe souhaitée et celles qui qualifient l'Europe condamnée. Les syntagmes associent à Europe (liste non exhaustive des occurrences attestées) :

- soit un adjectif qui modifie le référent en désignant moins l'institution que son projet politique (Europe sociale, Europe solidaire versus Europe libérale, Europe bureaucratique, Europe atlantiste)

- soit un complément du nom qui modifie le référent en délimitant son extension (Europe des citoyens, Europe des peuples, Europe de l'esprit, Europe des nations versus Europe de Bruxelles, Europe des patrons, Europe des économistes, Europe des puissants)

- soit un nom commun associé comme adjectif au nom propre dans un rapport de qualification -«qui est une» (Europe Lumière, Europe puissance versus Europe passoire, Europe forteresse).

Les discours populistes évoqués préalablement peuvent ainsi mobiliser une surdétermination adjectivale quand il s'agit de désigner l'Europe. Dans l'extrait suivant, même en l'absence de modalisation autonymique, les segments nominaux ( $\mathrm{Npr}+$ adjectif) sont attribués par le journaliste à la première dirigeante du Front national :

(7) Comme la présidente du FN [M. Le Pen] ne peut pas s'en prendre au monde entier, elle zoome sur plus petit : l'Europe, source de tous nos malheurs. L'Europepassoire nourrit les flots de l'immigration. L'Europe libérale tue notre économie. L'Europe tolérante est le paradis des djihadistes. L'Europe est le cheval de Troie d'une mondialisation que la France se doit, pour être fidèle à elle-même, de tenir à distance. Ce qui devrait lui permettre d'ériger des barrières douanières, de financer la retraite à 60 ans, de multiplier le nombre de fonctionnaires, d'augmenter le smic et les prestations sociales. Bref, de ramener le pays au temps des «trente glorieuses » (Le Monde, 18-12-2015).

Au-delà de l'attribution causale introductive (l'Europe, source de tous nos malheurs), les trois désignations suivantes associent à l'acte de nomination une caractérisation du référent. Europe-passoire, Europe libérale et Europe tolérante (et aussi l'attribut "cheval de Troie») prennent leur sens dans la situation de communication, ici reproduite par le journaliste, qui associe Marine Le Pen et ses électeurs potentiels. C'est dans ce cadre, c'est-à-dire en contexte, que l'axiologie négative associée à l'adjectif «libérale " et, plus encore, à l'adjectif «tolérante» s'impose. Condamner l'Europe tolérante, c'est entrer en relation dialogique et prendre position vis-à-vis d'autres locuteurs qui mobilisent ce même syntagme pour en appeler à la solidarité avec les réfugiés syriens. La référence, par Marine Le Pen, à ce qui est dit ailleurs participe ici du dialogisme de la nomination en même temps qu'elle participe à établir une relation de connivence avec son public. 


\subsection{Le nom propre modifié au service de la mobilisation}

24 l'unicité du référent «indépendamment de toute variation des univers de croyances ». Il s'associe invariablement au nom afin de marquer une "indépendance de la relation entre le nom propre et le référent par rapport à l'acte d'énonciation " (1994:228). L'article défini peut pourtant laisser place à un article indéfini présupposant une lecture fragmentée de l'entité Europe. Cet usage fréquent s'inscrit le plus souvent dans une visée persuasive. $\mathrm{Si}$, généralement, l'article indéfini suppose le prélèvement dans un ensemble de l'un de ses membres, par opposition au défini qui présuppose l'unicité du référent, l'indéfini peut également impliquer qu'une même entité peut être vue sous différents aspects. Il contribue alors à exhiber une manière d'être (ce que l'Union européenneest) par rapport à d'autres possibles (ce que l'Union européenne devrait être) et à confronter jugement de fait et jugement de valeur. Un effet rhétorique récurrent repose sur la mise en parallèle de deux désignations auxquelles le locuteur accorde une valeur axiologique opposée. C'est ainsi que l'alternative Europe sociale et Europe politique devient le cadre interprétatif privilégié de la crise économique et politique grecque aussi bien dans le discours rapporté des acteurs du conflit (8) que dans un commentaire journalistique dépourvu de modalisation autonymique (9) :

(8) «Dans cet affrontement entre une Europe sociale et une Europe libérale, nous n'avons pas trouvé d'alliés ", confie un membre du gouvernement grec. «C'est donc aux peuples d'Europe que nous en appelons pour changer le rapport de forces » (Le Monde, 3-07-2015).

(9) Ce qui s'est passé en Grèce, c'est le résultat d'une opposition entre deux visions différentes de l'Europe : d'un côté une Europe libérale, d'austérité ; de l'autre, une Europe sociale, une Europe des droits et des libertés (Le Monde, 7-07-2015).

La subordination de l'adjectif épithète au nom Europe impose l'indéfini. De l'article défini à l'indéfini, l'individuation par le nom propre disparaît en partie pour se rapprocher du nom commun (sans pour autant en revêtir les spécificités). L'extension adjectivale participe à une remotivation des termes: il ne s'agit plus de désigner l'Union européenne mais des projets politiques par rapport auxquels se positionne le locuteur. Ces désignations, idéologiquement investies, circulent dans l'espace médiatique et alimentent le propos sur l'Union européenne. L'opposition Europe libérale / Europe sociale, comme en (8) et (9) condense ainsi deux projets politiques dont les désignations en discours sont plus évocatrices et mobilisatrices que politiquement déterminées. Par leur récurrence et leur quasi-figement, sans doute peut-on rapprocher ces segments des « dénominations propres » définies par Veniard pour rendre compte du fonctionnement sémantico-référentiel des noms de guerre (2009: 63).

\section{Le retour du sens civilisationnel}

L'actualisation du sens d'Europe est plus frappante dans des désignations qui profitent d'une fréquence moins importante. Avec «Europe chrétienne» par exemple, la dénomination associe à la géographie non pas l'institution et un projet politique, mais une identité culturelle et religieuse. C'est ainsi le cas dans une tribune publié dans Le Figaro par la traditionaliste Christine Boutin peu avant les élections européennes de 2015:

Argumentation et Analyse du Discours, 17 | 2016 
(10) Je ne crois pas à une éthique européenne faisant du divorce de masse et de l'avortement en série l'horizon de notre politique familiale. Je rejette l'Europe Femen pour lui préférer décidément une Europe chrétienne. C'est pourquoi je pense que ces élections sont le moment d'un grand choix. Voulons-nous que l'Europe cesse d'être chrétienne ou non ? Voulons-nous que la déferlante migratoire et le règne du relativisme finissent de déraciner les riches heures de notre histoire ? Si nous laissons faire, la politique hors-sol et la barbarie triompheront avec le changement de civilisation promu par nos adversaires (Le Figaro, 22-04-2015). de l'invasion (cliché traditionnel du discours de l'extrême droite ici appuyé par 
l'argument quantitatif) associe à la géographie la rhétorique du choc des civilisations : renforcer les frontières, c'est préserver l'Europe comme civilisation.

En (10), (11) et (12), la dénomination Europe comme référent civilisationnel répond à une logique identitaire qui se déploie dans une opposition à l'autre. Est-ce à dire que cette valeur accordée au nom propre est une spécificité des discours de la droite et de l'extrême-droite?

Une attention portée au traitement médiatique des événements ukrainiens ${ }^{10}$ démontre qu'une telle conclusion serait réductrice. En nommant Europe l'utopie poursuivie par les manifestants, les journalistes partisans de l'intégration européenne trouvent une formidable opportunité événementielle pour relayer un discours devenu, depuis plusieurs années, presque inaudible en France au regard de la montée de l'euroscepticisme. L'événement devient l'objet d'un enjeu stratégique de positionnement politique : réhabiliter l'Europe. Ainsi, dans l'éditorial du Monde :

(13) Les démonstrations d'amour pour l'Union européenne sont suffisamment rares, ces temps-ci, pour que l'on s'y arrête. Absorbée par la crise de la dette, le combat pour la croissance et contre le chômage, la montée des populismes et la gestion de son élargissement, l'Europe a oublié qu'elle demeure une formidable force d'attraction. Pour des peuples qui ne bénéficient pas de l'Etat de droit, l'Europe symbolise l'espoir de la liberté, de la démocratie et de la modernité. C'est le message que nous adressent les dizaines de milliers d'Ukrainiens pro-européens (Le Monde, 02-12-2013)

Une large part des médias français met en scène l'attachement des "pro-européens » au socle de valeurs qui a présidé à la construction européenne au lendemain de la Seconde guerre mondiale. Ainsi Jacques Julliard dans son éditorial du 2 décembre 2013 confronte l'euroscepticisme actuel des citoyens au sein des Etats artisans historiques de l'intégration aux acteurs du conflit ukrainien :

(14) $\mathrm{Au}$ moment où les peuples européens, ces enfants gâtés de l'histoire contemporaine, font la fine bouche à l'égard de leur maison commune, c'est-à-dire l'Europe, c'est au nom de l'Europe, sous le drapeau de l'Europe, que se font tuer à Kiev les combattants de la liberté (Marianne, 02-12-2013).

En nommant Europe l'idéal poursuivi par les manifestants de Maïdan (voilant ainsi la très grande diversité idéologique de ces derniers), une partie de la presse française contribue à une requalification ethnocentrée du conflit ukrainien qui lui permet de réactualiser le discours des artisans historiques de l'intégration: la paix comme premier bien commun européen (Bellanger 2015). Des liens dialogiques, entre discours présents et passés, sont ainsi tissés par les commentateurs pour rappeler « de quoi Europe fut le nom ». Ce faisant, les commentateurs participent à une réhabilitation du mot par épuration de ses connotations négatives contemporaines. La nomination associe, dans une visée de (re-)légitimation, l'institution, le projet et la civilisation :

(15) L'Ukraine incarne pour l'Ouest un message propre à insuffler une énergie nouvelle capable de réveiller l'Europe de son sommeil : son histoire récente prouve que l'idée européenne ne s'arrête plus aux frontières administratives de l'Union, mais, plus à l'Est. Elle trace la ligne de partage entre civilisation et barbarie, entre dignité et servitude [rubrique Opinion, Tetyana Ogarkova, journaliste et politologue, Le Monde, 19-09-2014].

Dans une longue tribune, reprise d'un discours à Kiev tenu la veille, le philosophe Bernard Henri-Levy reprend à son compte l'opposition Europe/Russie. L'emphase participe là-aussi à une volonté de réhabiliter l'Europe en jouant sur la polyréférentialité du nom propre, l'institution devenant garante d'un socle de valeurs : 
(16) Vous avez, peuple du Maïdan, un rêve qui vous unit - et votre rêve, c'est l'Europe. [...] Car rien n'est plus concret que l'Europe que m'ont dépeinte, chaque fois, les femmes et hommes que vous avez portés à la tête de votre mouvement une Europe qui, pour toutes et tous, signifie liberté, juste gouvernance, lutte contre l'Etat-voyou des oligarques, citoyenneté. Vous donnez un sens, non pas «plus pur » comme a dit un poète français, mais plus précis, et plus riche, au mot d'Europe. C'est pourquoi je pense que la vraie Europe est ici. C'est pourquoi les vrais Européens, c'est ici, sur le Maïdan, qu'ils se trouvent réunis. C'est pourquoi l'Ukraine n'est pas ce vassal de l'empire russe mendiant son rattachement à l'Europe : elle est, en tout cas à cette heure, le cœur battant du continent (Le Monde, 10-02-2014). une proposée par le quotidien Libération concrétise l'interprétation partagée au sein d'une large part de l'espace médiatique français. La photographie, pleine page, représentant un homme armé en uniforme, est barrée d'un titre imposant par sa taille et le choix des mots : "Ukraine. Guerre contre l'Europe ». La formulation, concise et dépourvue de forme verbale, rapproche "Guerre contre l'Europe " du "désignant d'événement » (Calabrese 2013). Pour autant, cette désignation ne se fige pas et ne peut être assimilée à un "nom propre d'événement " puisqu'il n'y a pas de relation durable entre le signe et le référent (Krieg-Planque 2009). Ce que l'on retient, c'est que la désignation adoptée en une de Libération rend moins compte de l'événement pointé que de l'interprétation, naturellement subjective, adoptée par l'instance journalistique pour le nommer. "L'enjeu désignatif» (Véniard 2007) est double. Le nom commun classe l'événement dans la catégorie «guerre»- premier enjeu désignatif; le complément contribue à en fixer le sens - second enjeu désignatif. Ici, la référence à l'institution politique s'efface, en partie seulement, au profit d'une référence à un ensemble de valeurs censées fonder une civilisation ou, du moins, une identité européenne dont est, de fait, exclue la Russie. En nommant Europe et la cible de la politique étrangère russe et la cause défendue par les manifestants, l'instance journalistique offre au récit de l'actualité une logique dichotomique efficace au sens où elle dessine un face à face Europe/Russie.

\section{Conclusion}

Si Europe désigne initialement une jeune princesse phénicienne revêtant ainsi les attributs du nom propre prototypique, le référent initial s'efface ensuite largement. En contexte médiatique, la nomination l'Europe revêt le plus souvent aujourd'hui une valeur toponymique et s'impose comme une catégorisation géographique à même de cartographier les événements du monde. Cette valeur spatiale attribuée à l'Europe ne disparaît jamais totalement des actes de nomination mais se trouve souvent associée à d'autres référents qui en modifient le sens.

Argumentation et Analyse du Discours, 17 | 2016 
Cette ambiguïté référentielle peut servir des discours au degré d'argumentativité très variable. Ainsi, l'ambigüité qui pèse sur la recherche du référent sous-jacent dans le cas des usages journalistiques de la métonymie n'est pas au service d'une stratégie d'influence; elle revêt néanmoins des enjeux importants dans la représentation des politiques européennes en dissimulant la complexité des institutions et, par là même, des processus décisionnaires. La personnalisation, quant à elle, peut aussi bien servir un discours journalistique désubjectivisé qu'alimenter, dans le discours rapporté, une stratégie de stigmatisation de l'Europe. C'est en effet incarnée par le biais de la personnalisation que l'Europe peut se constituer comme adversaire politique et ainsi nourrir un discours de mobilisation.

A ce titre, le nom propre, dans le contexte du discours rapporté, s'inscrit dans un grand nombre de syntagmes nominaux où à la nomination s'associe la caractérisation. Quand un adjectif est subordonné au nom propre (impliquant la modification de ce dernier par l'indéfini), il participe à une remotivation des termes : l'institution s'efface au profit de projets politiques par rapport auxquels le locuteur se positionne soit pour les revendiquer soit pour s'y opposer. Ces désignations (une Europe sociale, une Europe citoyenne, une Europe libérale...) circulent et se répondent dans l'espace médiatique pour structurer le champ des revendications et des résistances à l'intégration européenne.

Enfin, en nous attachant spécifiquement aux commentaires journalistiques et politiques (discours rapportés) relatifs au conflit ukrainien, nous avons insisté sur la capacité de la nomination l'Europeà offrir un cadre interprétatif des événements à même de réhabiliter l'institution politique. L'acte de nomination associe en effet à l'institution souvent décriée le projet, la culture et la civilisation européenne. La polyréférentialité participe alors à l'actualisation du sens et à la réhabilitation du mot par épuration de ses connotations négatives.

Commentant les toponymes, Boyer et Paveau relèvent qu' «au-delà de la simple nomination de l'espace, et donc du domaine disciplinaire propre à l'onomastique, il existe des fonctionnements à teneur dialogique et polémique évidente " $(2008: 6)$. Autrement dit, comme dans le cas des noms de pays, des aspects à la fois géographiques, politiques et culturels concourent aux sens attribués à Europe.

\section{BIBLIOGRAPHIE}

Amossy, Ruth. 2014. Apologie de la polémique (Paris : PUF)

Aldrin, Philippe, Caroline Ollivier-Yanniv \& Jean-Michel Utard (éds). 2015. Les médiations de

l'Europe politique (Strasbourg : PUS)

Auboussier, Julien. 2015. « Les appels au boycott des élections européennes de 2014 et la mise à distance du discours institutionnel », Auboussier Julien \& Toni Ramoneda (éds). L'Europe en contre-discours (Besançon : PUFC), 129-145

Barthes, Roland, 1972 [1967], « Proust et les noms », Le degré zéro de l'écriture (Paris : Seuil) 
Bellanger, Marie-Eve. 2015. Territoires européens : discours et pratiques de l'élargissement (Genève : L'Harmattan-Academia)

Bonhomme, Marc. 1987. Linguistique de la métonymie (Berne : Peter Lang)

Boyer, Henri \& Marie-Anne Paveau. 2008. «Présentation », Mots 86, 5-8

Calabrese, Laura. 2013. L'événement en discours. Presse et mémoire sociale (Louvain-la-Neuve : Académia-L'Harmattan)

Charaudeau, Patrick. 2011. « Réflexions pour l'analyse du discours populiste », Mots 97, 101-116

Cislaru, Georgeta. 2005, «Etudes sémantiques et discursive du nom de pays dans la presse française ", thèse de doctorat en sciences du langage, Université de la Sorbonne Nouvelle Cislaru, Georgeta. 2008, « Le nom de pays comme outil de représentation sociale », Mots 86, 53-64 Develotte, Christine. 2000. « Titre de presse et représentation d'autrui en temps de crise », Mots 64, 23-38

Draga, Oana. 2010. «Pour une analyse décompositionnelle des noms propres toponymiques. Modèle de représentation sémantique », Synergies Roumanie 5, 177-193

Fiala, Pierre (éd.). 1993. «Europe/Allemagne. Réunification », Mots 34

Gary-Prieur, Marie-Noëlle, 2009, « Le nom propre, entre langue et discours », Les Carnets du Cediscor 11, 153-168

Gary-Prieur, Marie-Noëlle. 1994. Grammaire du nom propre (Paris : PUF)

Gosselin, André. 1995. «Les attributions causales dans la rhétorique politique », Hermès 16, 153-165

Guilhaumou, Jacques. 2015. «Provenance, émergence et invention du concept d'Europe. La généalogie historique au fondement de l'analyse du discours européen », Auboussier Julien \& Toni Ramoneda (éds). L'Europe en contre-discours (Besançon : PUFC), 17-35

Paveau, Marie-Anne. 2008. « Le toponyme, désignateur souple et organisateur mémoriel.

L'exemple du nom de bataille », Mots 86, 23-35

Hale, John. 1993. La civilisation de l'Europe à la Renaissance (Paris : Perrin)

Herman, Thierry \& Jufer Nicole. 2001. «L'éditorial, “vitrine idéologique du journal” ? , Semen 13, $135-162$

Kleiber, George. 2001. « Remarques sur la dénomination », Cahiers de praxématique 36, 21-41

Kleiber, George. 1984. « Dénomination et relation dénominatives », Langages 76, 77-94

Koren, Roselyne. 2004. «Argumentation, enjeux et pratique de l' "engagement neutre" : le cas de l'écriture de presse », Semen 17, 19-40

Krieg-Planque, Alice. 2009. La notion de « formule » en analyse du discours (Besançon : PUFC)

Krieg-Planque, Alice. 2008. « La notion d' "observable en discours" ", Burger Michel (éd.). L'analyse linguistique des discours médiatiques. Entre sciences du langage et sciences de la communication (Québec : Nota Bene), 59-81

Lakoff, George \& Mark Johnson. 1985. Les métaphores de la vie quotidienne (Paris : Minuit)

Lecolle, Michelle, Marie-Anne Paveau \& Sandrine Reboul-Toure (éds). 2009. « Le nom propre en discours », Les Carnets du Cediscor 11 
Lecolle, Michelle. 2001a. « Métonymie dans la presse écrite : entre discours et langue », Tranel 34-35, 153-170

Lecolle, Michelle. 2001b. « Personnification et métonymie dans la presse. Comment les différencier », Semen 15, 97-112

Leroy, Sarah \& Paul Siblot. 2000. « L'antonomase entre nom propre et catégorie nominale ", Mots $63,89-104$

Leroy, Sarah (éd.). 2005. « Noms propres : la modification », Langue française 146

Leroy, Sarah. 2004. Le nom propre en français (Paris : Ophrys)

Moirand, Sophie. 2000. «Variations discursives dans deux situations contrastées de la presse ordinaire », Les Carnets du Cediscor 6, 45-62

Mortureux, Marie-Françoise. 1993. «Paradigmes désignationnels », Semen 8, 123-141

Noailly, Michèle. 1999. « La querelle des noms propres », Modèles linguistiques 39, 107-112

Piguet, Marie-France. 1993, « L'Europe des Européens chez le comte de saint Simon », Mots 34, 25-38

Richard, Arnaud, Agnès Steuckardt \& Giancarlo Luxardo. 2015. « Européiste dans le discours radical "anti-européiste" ", Auboussier Julien \& Toni Ramoneda (éds). L'Europe en contre-discours (Besançon : PUFC), 163-178

Siblot, Paul. 2001. « De la dénomination à la nomination. Les dynamiques de la signifiance nominale et le propre du nom », Cahiers de praxématique 36, 189-214

Tournier, Maurice. 2000. « Europe, terme et terre d'immigration », Mots 63, 141-146

Wilmet, Marc. 1995. « Pour en finir avec le nom propre », L'information grammaticale 1 (65), 3-11

Yasri-Labrique, Eléonore. 2008. « La Turquie, terre eurasiatique et république bicéphale. Ankara et Istanbul dans la presse ", Mots 86, 37-52

\section{NOTES}

1. Le nom propre prototypique ne peut s'appliquer qu'à un seul être ou objet ou à une catégorie d'êtres ou d'objets pris en particulier et, ce faisant, il individualise ce qu'il désigne (Wilmet 1995). Son étude est le fruit d'une importante littérature en linguistique dans laquelle sont discutées les propriétés sémantiques spécifiques des noms propres. Nous retenons la discussion entre tenants du nom propre vide de sens pour qui il n'a pas de signification particulière et se rattache à ce qu'il désigne par convention et tenants du nom propre riche de sens pour qui il résume un sens complexe. Des travaux ont permis de concilier les approches du nom propre vide et riche de sens en réduisant le sens du nom propre X à la dénomination « être appelé $\mathrm{N}$ » (Kleiber 1984); d'autres ont ensuite rappelé que le nom propre ne possède un sens qu'en discours, "comme nomination en acte " (Siblot et Leroy 2000). Pour un retour sur l'ensemble des théories du nom propre en discours voir Lecolle, Paveau et Reboul-Touré 2009.

2. Au $19^{\mathrm{e}}$ siècle, encore, Europe est synonyme de civilisation. Cette conception est illustrée par la formule d'Augustin Thierry, en 1817: «Où finit la civilisation, où commence la grossièreté inculte, là doivent être les remparts de l'Europe, les seuls remparts en Europe " (Dans l'industrie, 1817). Illustrée aussi par De la réorganisation de la société européenne (1814) de Saint-Simon dans lequel on lit: «Il est essentiel [...] de considérer l'espèce humaine comme divisée en plusieurs variétés. Certainement la première variété de l'espèce humaine est la variété Européenne, 
puisqu'elle s'est maintenue dans la partie du globe qui produit le plus de blé et qui contient le plus de fer ». Pour une étude de ces deux textes de Saint Simon voir Piguet 1993.

3. Un cas particulier pourrait être traité : l'absence de pronom dans les titres de presse dans la configuration courante du titre bisegmental articulé par deux points (exemple : Europe : la crise est-elle passée ? ; Europe : l'impasse politique). Privé de prédéterminant, Europe fonctionne comme un référent discursivement indéfini, comme un substantif privé de toute détermination situationnelle. Europe se transforme alors en une catégorie d'appréhension du monde social.

4. Peut-être n'est-il pas inutile de rappeler aux lecteurs éloignés des débats européens que les frontières de l'Union européenne ne recouvrent pas, loin s'en faut, les frontières admises du continent. Des pays européens restent étrangers à l'institution comme la Norvège, l'Islande, la Suisse, la Serbie, la Bosnie-Herzégovine...

5. L'ouvrage Les médiations de l'Europe politique (Aldrin et al. 2015) illustre parfaitement la diversité des acteurs et des pratiques qui conduisent à l'élaboration et à l'adoption des politiques publiques européennes. L'Europe n'existe que dans la diversité de ses arènes de discussion et des débats, publicisés ou non, qui s'y déploient.

6. Pour la distinction métonymie/personnification dans la presse écrite, se rapporter au test de différentiation proposé par Michelle Lecolle (2002 : 104-107).

7. Nous précisons là que nombre de leaders eurosceptiques européens de gauche comme de droite, dont Marine Le Pen depuis 2004, siègent au Parlement européen et peuvent ainsi contribuer aux politiques européennes par la suite condamnées.

8. L'action qui provoqua le plus de discours polémiques date de 2013 à l'occasion du renoncement du pape Benoît XVI : des militantes Femen pénètrent dans la cathédrale NotreDame à Paris avant de se dévêtir et de faire tinter des cloches alors exposées dans le lieu de culte. En 2015, elles sont jugées pour la détérioration des cloches et sont finalement relaxées.

9. En septembre 2015, lors de son passage sur le plateau de On n'est pas couché (émission à forte audience du service public), N. Morano qualifie la France de "pays de race blanche »: "Il faut garder un équilibre dans le pays, c'est-à-dire sa majorité culturelle. Nous sommes un pays judéochrétien, le général de Gaulle le disait, de race blanche qui accueille des personnes étrangères. Je n'ai pas envie que la France devienne musulmane ». N. Sarkozy demande ensuite aux instances du parti (Les Républicains) de retirer l'investiture de N. Morano aux élections régionales.

10. A partir de la fin du mois de novembre 2013, la place de l'Indépendance (Maïdan), à Kiev, devient l'épicentre des manifestations contre le président Vikhtor Ianoukovitch. L'étincelle est le refus de ce dernier de signer un accord économique avec l'Union européenne au profit d'une coopération commerciale renforcée avec le voisin russe. Reléguée dans l'agenda médiatique depuis la « Révolution orange » de 2004, l'actualité ukrainienne attire de nouveau l'attention des médias français à partir de l'hiver 2013.

\section{RÉSUMÉS}

L'article interroge la polyréférentialité et l'actualisation en discours du nom propre Europe en contexte médiatique. Il s'agit dans un premier temps d'interroger les enjeux de la polyréférentialité liés aux usages métonymiques routinisés du nom propre dans le récit de l'actualité ainsi que les enjeux pragmatiques de la personnalisation. Dans un second temps, l'attention est portée au discours rapporté et aux usages du nom propre modifié qui jouent de la 
polyréférentialité dans une visée argumentative et mobilisatrice. Dès lors, l'actualisation du sens est interrogée au regard des enjeux politiques soulevés.

Our paper deals with multireference and the actualization of the proper noun Europe in the context of the media. First, we analyze the stakes of multireference linked to both routine uses of metonymy in news telling as well as to the pragmatic issues of personalization. The paper then focuses on reported speech and on the uses of the "modified proper noun" which play on multireference in order to persuade and mobilize. In the last part of the paper, the actualization and the production of meaning are called into question in the light of their political context.

INDEX

Mots-clés : dénomination, Europe, métonymie, polyréférentialité, référence

Keywords : denomination, Europe, metonymy, multireference, reference

\section{AUTEUR}

JULIEN AUBOUSSIER

Université de Franche-Comté, ELLIADD-DTEPS 